International Journal of Pure and Applied Mathematics

Volume 84 No. 1 2013, 73-83

ISSN: 1311-8080 (printed version); ISSN: 1314-3395 (on-line version)

url: http://www.ijpam.eu

doi: http://dx.doi.org/10.12732/ijpam.v84i1.5

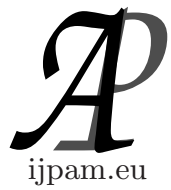

\title{
NEW UNDERESTIMATOR FOR MULTIVARIATE GLOBAL OPTIMIZATION WITH BOX CONSTRAINTS
}

\author{
Mohand Ouanes \\ Department of Mathematics \\ Mouloud Mammeri University \\ Tizi-Ouzou, 15000, ALGERIA
}

\begin{abstract}
The paper is concerned with the multivariate global optimization with box constraints. A new underestimator is investigated for twice continuously differentiable function on a box which is an extension of the approach developed in [5] for univariate global optimization.
\end{abstract}

AMS Subject Classification: 65K05, 90C30, 90C34

Key Words: global optimization, convex underestimator, exhaustive $w-$ subdivision, branch and bound

\section{Introduction}

We consider the following problem

$$
(P)\left\{\begin{array}{c}
\alpha:=\min f(s) \\
s \in H
\end{array}\right.
$$

where $H$ is a box in $R^{n}$ and $f: O \subset R^{n} \rightarrow R$ is twice continuously differentiable on an open convex set $O$ containing $H$. Even if constraints are simple, the problem remains very difficult to find the global optimum solution. In [1] the $\alpha \mathrm{BB}$ method consists in a construction of convex lower bound function of the functions of class $C^{2}$ which combines the objective function and the quadratic term, and uses the branch and bound algorithm. The review of principal methods

Received: October 30, 2012

(c) 2013 Academic Publications, Ltd. url: www.acadpubl.eu 
are presented in [2]. Efficient diagonal partitions are discussed in [6]. In [4] the interval method is used to compute global optimum. Basing on the idea developed in [5] for univariate global optimization we propose in this work a convex underestimating function for $f$ and a branch and bound algorithm for solving $(P)$. In our method we combine a multi-linear term with quadratic term to find a convex lower bound function. In our branch and bound algorithm, we solve at each iteration a convex problem with objective function as a polynomial. For branching, we use the exhaustive $w$-subdivision which has been shown to be efficient (see e.g. [5]). The paper is organized as follows: In Section 2 we present the main results. The algorithm and its convergence are presented in Section 3. Numerical examples found in the literature are treated in Section 4.

\section{Main Results}

Let $S:=\left[s_{0}, s_{1}\right]$ is a bounded closed interval in $R$ and $f$ is a continuously twice differentiable function on $S$ on which their second derivative is bounded, $h=s_{1}-s_{0}$ and $w_{j}: R \rightarrow R(j=0,1)$ the functions defined by

$$
\begin{aligned}
& w_{0}(s)=\left\{\begin{array}{cc}
\frac{s_{1}-s}{s_{1}-s_{0}} & \text { if } s_{0} \leq s \leq s_{1}, \\
0 & \text { otherwise }
\end{array}\right. \\
& w_{1}(s)=\left\{\begin{array}{cc}
\frac{s-s_{0}}{s_{1}-s_{0}} & \text { if } s_{0} \leq s \leq s_{1}, \\
0 & \text { otherwise }
\end{array}\right.
\end{aligned}
$$

Clearly,

$$
\sum_{i=1}^{1} w_{i}(s)=1, \forall s \in\left[s_{0}, s_{1}\right] \text { and } w_{i}\left(s_{j}\right)=0 \text { if } i \neq j, 1, \text { otherwise. }
$$

Let $L_{h} f$ be the piecewise linear interpolant to $f$ at points $s_{0}, s_{1}$.

$$
L_{h} f(s)=\sum_{i=0}^{1} f\left(s_{i}\right) w_{i}(s)
$$

In [5] we proposed a quadratic underestimator of $f$ on the interval

$$
L B(s)=L_{h} f(s)-\frac{1}{2} K\left(s-s_{0}\right)\left(s_{1}-s\right)
$$

where $K$ is positive number such that $\left|f^{\prime \prime}(s)\right| \leq K, \forall s \in\left[s_{0}, s_{1}\right]$. 
We can generalize this result to multivariate global optimization problem $(P)$. Let $H$ be the box $\Pi_{i=1}^{n}\left[s_{i}^{0}, s_{i}^{1}\right]$ whose vertex set is denoted $V(H)$. An element in $V(H)$ is denoted as $s:=\left(s_{1}^{i_{1}}, \ldots, s_{n}^{i_{n}}\right)$ with $i_{k}=0$ or 1 , for $k=1, \ldots, n$. We express $(P)$ in the form

$$
(P)\left\{\begin{array}{c}
\min f\left(s_{1}, \ldots, s_{n}\right) \\
\left(s_{1}, \ldots, s_{n}\right) \in H
\end{array}\right.
$$

and define the next function $\varphi: R^{n} \rightarrow R$ as

$$
\begin{gathered}
\varphi\left(s_{1}, \ldots, s_{n}\right)=L_{h_{n}}\left(\ldots\left(L_{h_{1}} f\left(s_{1}, \ldots, s_{n}\right)\right) \ldots\right)-\frac{1}{2} K\left(\sum_{i=1}^{n}\left(s_{i}-s_{i}^{0}\right)\left(s_{i}^{1}-s_{i}\right)\right) \\
=\sum_{i_{n}=0}^{1}\left(\ldots\left(\sum_{i_{1}=0}^{1} f\left(s_{1}^{i_{1}}, \ldots, s_{n}^{i_{n}}\right) w_{i_{1}}\left(s_{1}\right)\right) \ldots\right) w_{i_{n}}\left(s_{n}\right)-\frac{1}{2} K\left(\sum_{i=1}^{n}\left(s_{i}-s_{i}^{0}\right)\left(s_{i}^{1}-s_{i}\right)\right)
\end{gathered}
$$

where $K$ is a positive number such that

$$
K \geq\left\|H_{f}\left(s_{1}, \ldots, s_{n}\right)\right\|, \forall\left(s_{1}, \ldots, s_{n}\right) \in H,
$$

and $H_{f}\left(s_{1}, \ldots, s_{n}\right)$ is the Hessian matrix of the function $f$. Here the matrix norm of a $A=\left(a_{i j}\right)$ is defined by

$$
\|A\|:=\max _{i=1, \ldots, n} \sum_{j=1}^{n}\left|a_{i j}\right| .
$$

Theorem 2.1. i) The functions $\varphi$ and $f$ agree on the vertex set $V(H)$ of $H$.

ii) $\varphi$ is a minorization of $f$ on $H$, say $\varphi(s) \leq f(s), \forall s \in H$ if $K \geq$ $\max _{s \in H}\left|f_{s_{i} s_{i}}^{\prime \prime}(s)\right|$.

Proof. i) By the definition

$$
\begin{aligned}
& \varphi(s):= \\
& \quad \sum_{i_{n}=0}^{1}\left(\ldots\left(\sum_{i_{1}=0}^{1} f\left(s_{1}^{i_{1}}, \ldots, s_{n}^{i_{n}}\right) w_{i_{1}}\left(s_{1}\right)\right) \ldots\right) w_{i_{n}}\left(s_{n}\right)-\frac{1}{2} K\left(\sum_{i=1}^{n}\left(s_{i}-s_{i}^{0}\right)\left(s_{i}^{1}-s_{i}\right)\right) .
\end{aligned}
$$

Let $\left(s_{1}^{j_{1}}, \ldots, s_{n}^{j_{n}}\right)$ be a vertex of $H$. We have

$$
\varphi\left(s_{1}^{j_{1}}, \ldots, s_{n}^{j_{n}}\right)=
$$




$$
\sum_{i_{n}=0}^{1}\left(\ldots\left(\sum_{i_{1}=0}^{1} f\left(s_{1}^{i_{1}}, \ldots, s_{n}^{i_{n}}\right) w_{i_{1}}\left(s_{1}^{j_{1}}\right)\right) \ldots\right) w_{i_{n}}\left(s_{n}^{j_{n}}\right)-\frac{1}{2} K\left(\sum_{i=1}^{n}\left(s_{i}^{j_{i}}-s_{i}^{0}\right)\left(s_{i}^{1}-s_{i}^{j_{i}}\right)\right) .
$$

Clearly,

$$
\frac{1}{2} K\left(\sum_{i=1}^{n}\left(s_{i}^{j_{i}}-s_{i}^{0}\right)\left(s_{i}^{1}-s_{i}^{j_{i}}\right)\right)=0 .
$$

On the other hand, from the definition of $w_{i_{j}}\left(i_{j}=0,1\right)$ it follows that

$$
w_{0}\left(s_{1}^{0}\right)=w_{1}\left(s_{1}^{1}\right)=1 \text { and } w_{0}\left(s_{1}^{1}\right)=w_{1}\left(s_{1}^{0}\right)=0
$$

therefore

$$
\sum_{i_{1}=0}^{1} f\left(s_{1}^{i_{1}}, \ldots, s_{n}^{i_{n}}\right) w_{i_{1}}\left(s_{1}^{j_{1}}\right)=f\left(s_{1}^{j_{1}}, s_{2}^{i_{2}}, \ldots, s_{n}^{i_{n}}\right)
$$

Likewise

$$
\sum_{i_{2}=0}^{1} f\left(s_{1}^{j_{1}}, \ldots, s_{n}^{i_{n}}\right) w_{i_{2}}\left(s_{2}^{j_{2}}\right)=f\left(s_{1}^{j_{1}}, s_{2}^{j_{2}}, s_{3}^{i_{3}}, \ldots, s_{n}^{i_{n}}\right)
$$

and so on

$$
\sum_{i_{n}=0}^{1} f\left(s_{1}^{j_{1}}, \ldots, s_{n}^{i_{n}}\right) w_{i_{n}}\left(s_{n}^{j_{n}}\right)=f\left(s_{1}^{j_{1}}, \ldots, s_{n}^{j_{n}}\right)
$$

Hence

$$
\varphi\left(s_{1}^{j_{1}}, \ldots, s_{n}^{j_{n}}\right)=f\left(s_{1}^{j_{1}}, \ldots, s_{n}^{j_{n}}\right) .
$$

ii) We have

$$
\begin{aligned}
& \left|f\left(s_{1}, s_{2}, \ldots, s_{n}\right)-\sum_{i_{n}=0}^{1}\left(\ldots\left(\sum_{i_{1}=0}^{1} f\left(s_{1}^{i_{1}}, s_{2}^{i_{2}}, \ldots, s_{n}^{i_{n}}\right) w_{i_{1}}\left(s_{1}\right)\right) \ldots\right) w_{i_{n}}\left(s_{n}\right)\right| \\
= & \mid\left(f\left(s_{1}, s_{2}, \ldots, s_{n}\right)-\sum_{i_{1}=0}^{1} f\left(s_{1}^{i_{1}}, s_{2}, \ldots, s_{n}\right) w_{i_{1}}\left(s_{1}\right)\right)+ \\
& \left(\sum_{i_{1}=0}^{1} f\left(s_{1}^{i_{1}}, s_{2}, \ldots, s_{n}\right) w_{i_{1}}\left(s_{1}\right)-\sum_{i_{2}=0}^{1}\left(\sum_{i_{1}=0}^{1} f\left(s_{1}^{i_{1}}, s_{2}^{i_{2}}, \ldots, s_{n}\right) w_{i_{1}}\left(s_{1}\right)\right) w_{i_{2}}\left(s_{2}\right)\right)+ \\
& \left(\sum_{i_{2}=0}^{1}\left(\sum_{i_{1}=0}^{1} f\left(s_{1}^{i_{1}}, s_{2}^{i_{2}}, \ldots, s_{n}\right) w_{i_{1}}\left(s_{1}\right)\right) w_{i_{2}}\left(s_{2}\right)-\ldots\right)+\ldots+ \\
& \left(\sum_{i_{n-1}=0}^{1}\left(\ldots\left(\sum_{i_{1}=0}^{1} f\left(s_{1}^{i_{1}}, s_{2}^{i_{2}}, \ldots, s_{n-1}^{i_{n-1}}, s_{n}\right) w_{i_{1}}\left(s_{1}\right)\right) \ldots\right) w_{i_{n-1}}\left(s_{n-1}\right)-\right.
\end{aligned}
$$




$$
\begin{aligned}
& \left.\sum_{i_{n}=0}^{1}\left(\ldots\left(\sum_{i_{1}=0}^{1} f\left(s_{1}^{i_{1}}, s_{2}^{i_{2}}, \ldots, s_{n}^{i_{n}}\right) w_{i_{1}}\left(s_{1}\right)\right) \ldots\right) w_{i_{n}}\left(s_{n}\right)\right) \mid \\
\leq & \left|\left(f\left(s_{1}, s_{2}, \ldots, s_{n}\right)-\sum_{i_{1}=0}^{1} f\left(s_{1}^{i_{1}}, s_{2}, \ldots, s_{n}\right) w_{i_{1}}\left(s_{1}\right)\right)\right|+ \\
& \left|\sum_{i_{1}=0}^{1} f\left(s_{1}^{i_{1}}, s_{2}, \ldots, s_{n}\right) w_{i_{1}}\left(s_{1}\right)-\sum_{i_{2}=0}^{1}\left(\sum_{i_{1}=0}^{1} f\left(s_{1}^{i_{1}}, s_{2}^{i_{2}}, \ldots, s_{n}\right) w_{i_{1}}\left(s_{1}\right)\right) w_{i_{2}}\left(s_{2}\right)\right|+ \\
& \left|\sum_{i_{2}=0}^{1}\left(\sum_{i_{1}=0}^{1} f\left(s_{1}^{i_{1}}, s_{2}^{i_{2}}, \ldots, s_{n}\right) w_{i_{1}}\left(s_{1}\right)\right) w_{i_{2}}\left(s_{2}\right)-\ldots\right|+\ldots+ \\
& \mid \sum_{i_{n-1}=0}^{1}\left(\ldots\left(\sum_{i_{1}=0}^{1} f\left(s_{1}^{i_{1}}, s_{2}^{i_{2}}, \ldots, s_{n-1}^{i_{n-1}}, s_{n}\right) w_{i_{1}}\left(s_{1}\right)\right) \ldots\right) w_{i_{n-1}}\left(s_{n-1}\right)- \\
& \left.\sum_{i_{n}=0}^{1}\left(\ldots\left(\sum_{i_{1}=0}^{1} f\left(s_{1}^{i_{1}}, s_{2}^{i_{2}}, \ldots, s_{n}^{i_{n}}\right) w_{i_{1}}\left(s_{1}\right)\right) \ldots\right) w_{i_{n}}\left(s_{n}\right)\right) \mid \\
\leq & \frac{1}{2} K\left(s_{1}-s_{1}^{0}\right)\left(s_{1}^{1}-s_{1}\right)+\frac{1}{2} K\left(s_{2}-s_{2}^{0}\right)\left(s_{2}^{1}-s_{2}\right)+\ldots+\frac{1}{2} K\left(s_{n}-s_{n}^{0}\right)\left(s_{n}^{1}-s_{n}\right) \\
= & \frac{1}{2} K\left(\sum_{i=1}^{n}\left(s_{i}-s_{i}^{0}\right)\left(s_{i}^{1}-s_{i}\right)\right)
\end{aligned}
$$

Thus

$$
\varphi(s) \leq f(s), \forall s \in H .
$$

As an immediate consequence of this theorem we have

$$
\begin{aligned}
\mid \sum_{i_{n}=0}^{1}\left(\ldots \left(\sum_{i_{1}=0}^{1} f\left(s_{1}^{i_{1}}, \ldots, s_{n}^{i_{n}}\right)\right.\right. & \left.\left.w_{i_{1}}\left(s_{1}\right)\right) \ldots\right) w_{i_{n}}\left(s_{n}\right)-f\left(s_{1}, \ldots, s_{n}\right) \mid \\
\leq & \frac{1}{2} K\left(h_{1}^{2}+\ldots+h_{n}^{2}\right) \quad \text { with } h_{i}=s_{i}^{1}-s_{i}^{0} .
\end{aligned}
$$

Theorem 2.2. The function $\varphi$ is convex on $H$ if

$$
K \geq \max _{s \in H} \max _{i=1, \ldots, n} \sum_{j=1, j \neq i}^{n}\left|f_{s_{i} s_{j}}^{\prime \prime}(s)\right| .
$$


Proof. We express $\varphi$ in the form

$$
\begin{aligned}
\varphi(s)= & \sum_{i_{n}=0}^{1}\left(\ldots\left(\sum_{i_{1}=0}^{1} f\left(s_{1}^{i_{1}}, \ldots, s_{n}^{i_{n}}\right) w_{i_{1}}\left(s_{1}\right)\right) \ldots\right) w_{i_{n}}\left(s_{n}\right)-\frac{1}{2} K\left(\sum_{i=1}^{n}\left(s_{i}-s_{i}^{0}\right)\left(s_{i}^{1}-s_{i}\right)\right) \\
= & \sum_{i_{n}=0}^{1}\left(\ldots\left(\sum_{i_{1}=0}^{1} f\left(s_{1}^{i_{1}}, \ldots, s_{n}^{i_{n}}\right) w_{i_{1}}\left(s_{1}\right)\right) \ldots\right) w_{i_{n}}\left(s_{n}\right) \\
& +\frac{1}{2} K s_{i}^{2}-\frac{1}{2} K \sum_{i=1}^{n}\left(\left(s_{i}^{1}+s_{i}^{0}\right) s_{i}-s_{i}^{0} s_{i}^{1}\right) .
\end{aligned}
$$

Since the part

$$
\frac{1}{2} K \sum_{i=1}^{n}\left(\left(s_{i}^{1}+s_{i}^{0}\right) s_{i}-s_{i}^{0} s_{i}^{1}\right)
$$

is linear, it suffices to prove that the function $\Phi$ defined by

$$
\Phi\left(s_{1}, \ldots, s_{n}\right):=\sum_{i_{n}=0}^{1}\left(\ldots\left(\sum_{i_{1}=0}^{1} f\left(s_{1}^{i_{1}}, \ldots, s_{n}^{i_{n}}\right) w_{i_{1}}\left(s_{1}\right)\right) \ldots\right) w_{i_{n}}\left(s_{n}\right)+\frac{1}{2} K s_{i}^{2}
$$

is convex. This amounts to show that the Hessian matrix of $\Phi$, denoted $H_{\Phi}$, is semi-definite positive. Let

$$
L_{h} f\left(s_{1}, \ldots, s_{n}\right):=\sum_{i_{n}=0}^{1}\left(\ldots\left(\sum_{i_{1}=0}^{1} f\left(s_{1}^{i_{1}}, \ldots, s_{n}^{i_{n}}\right) w_{i_{1}}\left(s_{1}\right)\right) \ldots\right) w_{i_{n}}\left(s_{n}\right) .
$$

From the definition of $w_{i_{j}}$, it is easy to see that all elements $\left(L_{h} f\right)_{s_{i} s_{i}}^{\prime \prime}$ are zero, for $i=1, \ldots, n$. Hence $H_{\Phi}$ takes the form

$$
H_{\Phi}=\left(\begin{array}{ccccc}
K & L_{12} & \cdot & \cdot & L_{1 n} \\
L_{21} & K & L_{23} & \cdot & L_{2 n} \\
\cdot & \cdot & \cdot & \cdot & \cdot \\
\cdot & \cdot & \cdot & \cdot & \cdot \\
L_{n 1} & \cdot & \cdot & \cdot & K
\end{array}\right)
$$

with $L_{i j}:=\left(L_{h} f\right)_{s_{i} s_{j}}^{\prime \prime}\left(s_{1}, \ldots, s_{n}\right)$ is the second mixed derivatives of $L_{h} f$ with respect to the variables $s_{i}$ and $s_{j}$.

The second mixed derivatives of $L_{h} f$, for example the second mixed derivative with respect to variables $s_{1}$ and $s_{2}$, can be computed as follows. First, the derivative with respect to one variable, for example, $s_{1}$, can be expressed as

$$
L_{h} f_{s_{1}}^{\prime}\left(s_{1}, \ldots, s_{n}\right)=\left(\sum_{i_{n}=0}^{1}\left(\ldots\left(\sum_{i_{1}=0}^{1} f\left(s_{1}^{i_{1}}, \ldots, s_{n}^{i_{n}}\right) w_{i_{1}}\left(s_{1}\right)\right) \ldots\right) w_{i_{n}}\left(s_{n}\right)\right)_{s_{1}}^{\prime}
$$




$$
\begin{aligned}
= & \sum_{i_{n}=0}^{1}\left(\ldots\left(\sum_{i_{2}=0}^{1}\left(f\left(s_{1}^{0}, s_{2}^{i_{2}}, \ldots, s_{n}^{i_{n}}\right) w_{0}^{\prime}\left(s_{1}\right)+f\left(s_{1}^{1}, s_{2}^{i_{2}}, \ldots, s_{n}^{i_{n}}\right) w_{1}^{\prime}\left(s_{1}\right)\right) w_{i_{2}}\left(s_{2}\right)\right) \ldots\right) \\
& w_{i_{n}}\left(s_{n}\right) \\
= & \sum_{i_{n}=0}^{1}\left(\ldots\left(\sum_{i_{2}=0}^{1}\left(f\left(s_{1}^{0}, s_{2}^{i_{2}}, \ldots, s_{n}^{i_{n}}\right) \frac{-1}{s_{1}^{1}-s_{1}^{0}}+f\left(s_{1}^{1}, s_{2}^{i_{2}}, \ldots, s_{n}^{i_{n}}\right) \frac{1}{s_{1}^{1}-s_{1}^{0}}\right) w_{i_{2}}\left(s_{2}\right)\right) \ldots\right) \\
& w_{i_{n}}\left(s_{n}\right) \\
= & \sum_{i_{n}=0}^{1}\left(\ldots\left(\sum_{i_{2}=0}^{1}\left(\frac{f\left(s_{1}^{1}, s_{2}^{i_{2}}, \ldots, s_{n}^{i_{n}}\right)-\left(f\left(s_{1}^{0}, s_{2}^{i_{2}}, \ldots, s_{n}^{i_{n}}\right)\right.}{s_{1}^{1}-s_{1}^{0}}\right) w_{i_{2}}\left(s_{2}\right)\right) \ldots\right) w_{i_{n}}\left(s_{n}\right) \\
= & \sum_{i_{n}=0}^{1}\left(\ldots\left(\sum_{i_{2}=0}^{1}\left(f_{s_{1}}^{\prime}\left(\xi, s_{2}^{i_{2}}, \ldots, s_{n}^{i_{n}}\right)\right) w_{i_{2}}\left(s_{2}\right)\right) \ldots\right) w_{i_{n}}\left(s_{n}\right)
\end{aligned}
$$

with $s_{1}^{0}<\xi<s_{1}^{1}$.

Afterwards the second derivative with respect to the first two variables is computed as:

$$
\begin{aligned}
& \left.L_{h} f_{s_{1} s_{2}}^{\prime \prime}\left(s_{1}, \ldots, s_{n}\right)=\sum_{i_{n}=0}^{1}\left(\ldots\left(\sum_{i_{2}=0}^{1}\left(f_{s_{1}}^{\prime}\left(\xi, s_{2}^{i_{2}}, \ldots, s_{n}^{i_{n}}\right)\right) w_{i_{2}}\left(s_{2}\right)\right) \ldots\right) w_{i_{n}}\left(s_{n}\right)\right)_{s_{2}}^{\prime} \\
& =\sum_{i_{n}=0}^{1}\left(\ldots \left(\sum _ { i _ { 3 } = 0 } ^ { 1 } \left(f_{s_{1}}^{\prime}\left(\xi, s_{2}^{0}, s_{3}^{i_{3}}, \ldots, s_{n}^{i_{n}}\right) w_{0}^{\prime}\left(s_{2}\right)\right.\right.\right. \\
& \left.\left.\left.+f_{s_{1}}^{\prime}\left(\xi, s_{1}^{1}, s_{2}^{i_{2}}, \ldots, s_{n}^{i_{n}}\right) w_{1}^{\prime}\left(s_{2}\right)\right) w_{i_{3}}\left(s_{3}\right)\right) \ldots\right) w_{i_{n}}\left(s_{n}\right) \\
& =\sum_{i_{n}=0}^{1}\left(\ldots \left(\sum _ { i _ { 3 } = 0 } ^ { 1 } \left(f_{s_{1}}^{\prime}\left(\xi, s_{2}^{0}, s_{3}^{i_{3}}, \ldots, s_{n}^{i_{n}}\right) \frac{-1}{s_{2}^{1}-s_{2}^{0}}\right.\right.\right. \\
& \left.\left.\left.+f_{s_{1}}^{\prime}\left(\xi, s_{1}^{1}, s_{2}^{i_{2}}, \ldots, s_{n}^{i_{n}}\right) \frac{1}{s_{2}^{1}-s_{2}^{0}}\right) w_{i_{3}}\left(s_{3}\right)\right) \ldots\right) w_{i_{n}}\left(s_{n}\right) \\
& =\sum_{i_{n}=0}^{1}\left(\ldots\left(\sum_{i_{3}=0}^{1}\left(\frac{f_{s_{1}}^{\prime}\left(\xi, s_{1}^{1}, s_{2}^{i_{2}}, \ldots, s_{n}^{i_{n}}\right)-f_{s_{1}}^{\prime}\left(\xi, s_{2}^{0}, s_{3}^{i_{3}}, \ldots, s_{n}^{i_{n}}\right)}{s_{2}^{1}-s_{2}^{0}}\right) w_{i_{3}}\left(s_{3}\right)\right) \ldots\right) w_{i_{n}}\left(s_{n}\right) \\
& =\sum_{i_{n}=0}^{1}\left(\ldots\left(\sum_{i_{3}=0}^{1}\left(f_{s_{1} s_{2}}^{\prime \prime}\left(\xi, \eta, s_{3}^{i_{3}}, \ldots, s_{n}^{i_{n}}\right)\right) w_{i_{3}}\left(s_{3}\right)\right) \ldots\right) w_{i_{n}}\left(s_{n}\right)
\end{aligned}
$$

with $s_{1}^{0}<\xi<s_{1}^{1}$ and $s_{2}^{0}<\eta<s_{2}^{1}$.

Since

$$
w_{0}(x)+w_{1}(x)=1 \text { for all } x \in\left[s_{i}^{0}, s_{i}^{1}\right], i=1, \ldots, n
$$


we have

$$
\sum_{i_{3}=0}^{1} f_{s_{1} s_{2}}^{\prime \prime}\left(\xi, \eta, s_{3}^{i_{3}}, \ldots, s_{n}^{i_{n}}\right) w_{i_{3}}\left(s_{3}\right) \leq \max _{s_{3} \in\left[s_{3}^{0}, s_{3}^{1}\right]} f_{s_{1} s_{2}}^{\prime \prime}\left(\xi, \eta, s_{3}, \ldots, s_{n}^{i_{n}}\right),
$$

and so on

$$
\left.\sum_{i_{n}=0}^{1}\left(\ldots\left(\sum_{i_{3}=0}^{1} f_{s_{1} s_{2}}^{\prime \prime}\left(\xi, \eta, s_{3}^{i_{3}}, \ldots, s_{n}^{i_{n}}\right) w_{i_{3}}\left(s_{3}\right)\right) \ldots\right) w_{i_{n}}\left(s_{n}\right)\right) \leq \max _{\left(s_{1}, \ldots, s_{n}\right) \in H} f_{s_{1} s_{2}}^{\prime \prime}\left(s_{1}, \ldots, s_{n}\right) .
$$

Hence

$$
\left|L_{h} f_{s_{1} s_{2}}^{\prime \prime}\left(s_{1}, \ldots, s_{n}\right)\right| \leq \max _{\left(s_{1}, \ldots, s_{n}\right) \in H}\left|f_{s_{1} s_{2}}^{\prime \prime}\left(s_{1}, \ldots, s_{n}\right)\right| .
$$

Likewise, for any pair $i \neq j$ we have

$$
\left|\left(L_{h} f\right)_{s_{i} s_{j}}^{\prime \prime}\right| \leq \max _{\left(s_{1}, \ldots, s_{n}\right) \in H}\left|f_{s_{i} s_{j}}^{\prime \prime}\left(s_{1}, \ldots, s_{n}\right)\right| .
$$

Consequently, if

$$
K \geq \max _{s \in H} \max _{i=1, \ldots, n} \sum_{j=1, j \neq i}^{n}\left|f_{s_{i} s_{j}}^{\prime \prime}(s)\right|
$$

then

$$
K \geq \max _{s \in H} \max _{i=1, \ldots, n} \sum_{j=1, j \neq i}^{n}\left|L_{h} f_{s_{i} s j}^{\prime \prime}\left(s_{1}, \ldots, s_{n}\right)\right|
$$

and therefore $H_{\Phi}$ is semi-definite positive.

\section{Notes and Comments}

The inequality $K \geq \max _{s \in H} \max _{i=1, \ldots, n} \sum_{j=1, j \neq i}^{n}\left|f_{s_{i} s_{j}}^{\prime \prime}(s)\right|$ implies that $K \geq$ $\max _{s \in H}\left|f_{s_{i} s_{i}}^{\prime \prime}(s)\right|$, a sufficient condition for $\varphi$ to be a underestimator of $f$.

\section{A Branch and Bound Algorithm and its Convergence}

We can now describe the branch and bound algorithm for solving $(\mathrm{P})$. Denote by, $\mathrm{LB}_{k}, \mathrm{UB}_{k}$ and $s^{k}$ respectively the best lower bound, the best upper bound and the best solution to $(\mathrm{P})$ at iteration $\mathrm{k}$.

\section{Algorithm BB:}


- Initialization: Let $\varepsilon$ be a given sufficiently small number. Compute $K$, a upper bound of $\left\|H_{f}\right\|$ on $\mathrm{H}$. Set $T^{0}=H$, solve the convex program

$$
\min \left\{\varphi(s): s \in T^{k}\right\}
$$

to obtain an optimal solution $\widetilde{\mathbf{s}}^{0}$.

Set $U B_{0}:=\min \left\{\min _{s \in V(H)} f(s), f\left(\widetilde{\mathbf{s}}^{0}\right)\right\}, L B\left(T^{0}\right):=\varphi\left(\widetilde{\mathbf{s}}^{0}\right)$.

Let $s^{0}$ such that $U B_{0}=f\left(s^{0}\right)$ and $L B_{0}=L B\left(T^{0}\right)$.

If $U B_{0}-L B_{0} \leq \varepsilon$ then STOP $s^{0}$ is an $\varepsilon$ - optimal solution,

else set $M \leftarrow\left\{T^{0}\right\}, \quad k \leftarrow 1$, and go to iteration k.

- Iteration $\mathrm{k}$

k1. Let $T^{k}=\prod_{k=1}^{n}\left[a_{k}, b_{k}\right] \in M$ be the rectangle such that $L B_{k}=$ $L B\left(T^{k}\right)$, and $\widetilde{\mathbf{s}}^{k}$ be the solution of Problem (3.1).

Set $K_{k}$ such that $\left\|H_{f}(s)\right\| \leq K_{k}, \forall s \in T^{k}$.

k2. Bisect $T^{k}$ into two subrectangles $T^{k 1}, T^{k 2}$ by w-subdivision procedure via $\widetilde{\mathbf{s}}^{k}$.

k3. For $i=1,2$ do

Solve the convex program (3.1) where $k$ is replaced by $k_{i}$ to obtain an optimal solution $\widetilde{\mathrm{s}}^{k i}$.

k4. Update the upper bound $U B_{k}=\min \left\{U B_{k}, f\left(\widetilde{\mathbf{s}}^{k 1}\right), f\left(\widetilde{\mathbf{s}}^{k 2}\right)\right\}$. Let $s^{k}$ be the best current solution, i.e. $f\left(s^{k}\right)=U B_{k}$.

Set $M \leftarrow M \cup\left\{T_{i}^{k}: L B\left(T_{i}^{k}\right)<U B^{k}-\varepsilon, i=1,2\right\} \backslash\left\{T^{k}\right\}$.

Update the lower bound: $L B_{k}=\min \{L B(T): T \in M\}$.

k5. If $M=\emptyset$ then STOP, $s^{k}$ is an optimal solution.

else set $k \leftarrow k+1$, and return to $\mathbf{k} \mathbf{1}$.

Theorem 3.1. Either the algorithm is finite or it generates a bounded sequence $\left\{s^{k}\right\}$ which converges to an optimal solution of $(P)$.

Proof. If the algorithm stops after a finite number of iterations then by the stopping rule the solution is optimal and exact.

If it generates an infinite sequence it suffices to show that $\lim _{k \rightarrow \infty}\left(U B_{k}-\right.$ $\left.L B_{k}\right)=0$.

We have 


$$
\begin{aligned}
& 0 \leq U B_{k}-L B_{k}=f\left(s^{k}\right)-\varphi\left(\widetilde{\mathbf{s}}^{k}\right) \leq f\left(\widetilde{\mathbf{s}}^{k}\right)-\varphi\left(\widetilde{\mathbf{s}}^{k}\right) \\
& \leq f\left(\widetilde{\mathbf{s}}^{k}\right)-\sum_{i_{n}=0}^{1}\left(\ldots\left(\sum_{i_{1}=0}^{1} f\left(s_{1}^{i_{1}}, \ldots, s_{n}^{i_{n}}\right) w_{i_{1}}\left(\widetilde{\mathrm{s}_{1}}{ }^{k}\right)\right) \ldots\right) w_{i_{n}}\left(\widetilde{\mathrm{s}_{\mathrm{n}}}{ }^{k}\right) \\
& +\frac{1}{2} K\left(\sum_{i=1}^{n}\left(\widetilde{\mathrm{s}_{\mathrm{i}}}{ }^{k}-s_{i}^{0 k}\right)\left(s_{i}^{1 k}-{\widetilde{\mathrm{s}_{\mathrm{i}}}}^{k}\right)\right) \\
& \leq f\left(\widetilde{\mathbf{s}}^{k}\right)-\min \sum_{i_{n}=0}^{1}\left(\ldots\left(\sum_{i_{1}=0}^{1} f\left(s_{1}^{i_{1}}, \ldots, s_{n}^{i_{n}}\right) w_{i_{1}}\left({\widetilde{\mathrm{s}_{1}}}^{k}\right)\right) \ldots\right) w_{i_{n}}\left({\widetilde{\mathrm{s}_{\mathrm{n}}}}^{k}\right) \\
& +\frac{1}{2} K\left(\sum_{i=1}^{n}\left(\widetilde{\mathrm{s}_{\mathrm{i}}^{k}}-s_{i}^{0 k}\right)\left(s_{i}^{1 k}-{\widetilde{\mathrm{s}_{\mathrm{i}}}}^{k}\right)\right) \leq \\
& f\left(\widetilde{\mathrm{s}}^{k}\right)-\min _{s \in V\left(T^{k}\right)} f(s)+\frac{1}{2} K\left(\sum_{i=1}^{n}\left({\widetilde{\mathrm{s}_{\mathrm{i}}}}^{k}-s_{i}^{0 k}\right)\left(s_{i}^{1 k}-{\widetilde{\mathrm{s}_{\mathrm{i}}}}^{k}\right)\right) \leq \\
& \delta\left\|\widetilde{\mathbf{s}}^{k}-\bar{s}^{k}\right\|+\frac{1}{2} K\left(h_{1 k}^{2}+\ldots+h_{n k}^{2}\right) \leq \delta\left(\left(h_{1 k}^{2}+\ldots+h_{n k}^{2}\right)\right)^{\frac{1}{2}}+\frac{1}{2} K\left(h_{1 k}^{2}+\ldots+h_{n k}^{2}\right)
\end{aligned}
$$

(i.e. by the continuity of $f(\delta>0)$ and by using the lengths of the edges of the hyperrectangle $\left.T^{k}\right)$.

The exhaustive $w-$ subdivision implies $\lim _{k \rightarrow \infty} h_{i k}=0, \forall i=1, \ldots, n$.

Hence $\operatorname{Lim}_{k \rightarrow \infty}\left(U B_{k}-L B_{k}\right)=0$ and the theorem is proved.

\section{Numerical Examples}

Example 1. (see [3]) $f\left(s_{1}, s_{2}=-\sin \left(s_{1}\right) \sin \left(s_{1} s_{2}\right),[0,4] \times[0,4], K=\right.$ $12, \varepsilon=10^{-5}$.

The solution found by our method is $(1.5588,0.99655)$ and

$$
f(1.5588,0.99655)=-0.99978)
$$

with practically the same precision as in [3], the number of function evaluations is 889 which is less than that of [3] which is equal to 1013.

Example 2. (see [3]) $f\left(s_{1}, s_{2}\right)=\left(s_{1}-2\right)^{2}+\left(s_{2}-1\right)^{2}+0.04\left(1-\frac{s_{1}^{2}}{4}-s_{2}^{2}\right)+$ $\frac{\left(s_{1}-2 s_{2}+1\right)^{2}}{0.2},[1,2] \times[1,2], K=80, \varepsilon=10^{-5}$.

The solution found by our method is $(1.7936,1.3772)$ and $f(1.7936,1.3772)=$ 0.16905 , with practically the same precision as in [3], the number of function 
evaluations is 805 which is significantly less than that of [3] which is equal to 2613.

\section{Conclusion}

We have proposed in this paper a new convex underestimator for twice continuously differentiable functions and a branch and bound algorithm for minimizing this functions with box constraints. The convergence of the algorithm is shown and numerical examples found in the literature are treated efficiently.

\section{References}

[1] C.S. Adjiman, I.P. Androulakis, C.A. Floudas, A global optimization method, $\alpha \mathrm{BB}$, for general twice-differentiable NLPs - II. Implementation and computational results, Comput. Chem. Eng., 22, No. 9 (1998), 11591179.

[2] C.A. Floudas, C.E. Gounaris, A review of recent advances in global optimization, J. Glob. Optim., (2008), doi: 10.1007/s10898-008-9332-8.

[3] E. Gourdin, B. Jaumard, R. Ellaia, Global optimization of Holder functions, J. of Global Optimization, 8, No. 4 (1996), 323-348.

[4] E. Hansen, Global optimization using Interval analysis, the multidimensionnal case, Numer. Math., 34 (1980), 247-270.

[5] Le Thi Hoai An, Ouanes Mohand, Convex quadratic underestimation and branch and bound for univariate global optimization with one nonconvex constraint, RAIRO Oper. Res., 40, No. 3 (2006), 285-302.

[6] D.Y. Sergeyev, D.E. Kvasov, Global search based on efficient diagonal partitions and a set of Lipschitz constants, SIAM J. Optim., 16, No. 3 (2006), 910-937. 
